\title{
Nonbinary Quasi-Cyclic LDPC-Based Coded Modulation for Beyond 100-Gb/s Transmission
}

\author{
Murat Arabaci, Ivan B. Djordjevic, Ross Saunders, and Roberto M. Marcoccia
}

\begin{abstract}
We propose using coded modulation based on nonbinary quasi-cyclic low-density parity-check (LDPC) codes for beyond 100-Gb/s optical transmission. The proposed scheme not only lowers the latency in the system but also offers much higher coding gains than its binary counterpart based on bit-interleaved coded modulation (BICM). We show that using component LDPC codes over high order finite fields and matching bandwidth-efficient modulations, our scheme can provide around 1-dB coding gain improvement at the bit-error ratio of $10^{-6}$ compared to a corresponding BICM-based scheme.
\end{abstract}

Index Terms-Forward-error correction, low-density paritycheck (LDPC) codes, optical communication, quasi-cyclic codes.

\section{INTRODUCTION}

$\mathbf{T}$ HE PAST two decades have witnessed a tremendous increase in transport capabilities of fiber-optic communication systems. One of the most important advancements enabling this increase was the emergence of dense wavelength-division multiplexing (DWDM) as a viable, flexible, and cost-effective transport technology. Today, $100 \mathrm{~Gb} / \mathrm{s}$ per DWDM channel transmission is envisioned by network operators. However, the cost of devices supporting such high data rates and the cost of their installation are still prohibitively high. Furthermore, the significant degradation in the performance of these systems due to several transmission impairments including fiber nonlinearities and polarization-mode dispersion (PMD) exacerbates the problem.

Coded modulation techniques can be employed to address the aforementioned challenges simultaneously. The underlying idea is to map each $m$-bit block to a nonbinary symbol, and transmit nonbinary symbols instead of bits per channel use. This, in effect, reduces the symbol rate at which transmission, detection, and decoding are performed to the rates at which devices readily installed in today's fiber-optic networks operate. In addition to being more practical, systems operating at lower symbol rates lend themselves to comparably easier nonlinearity and PMD management.

\footnotetext{
Manuscript received September 14, 2009; revised December 15, 2009; accepted January 08, 2010. First published February 02, 2010; current version published February 26, 2010. This work was supported in part in part by Opnext, Inc., and in part by the National Science Foundation (NSF) under Grant IHCS-0725405.

M. Arabaci and I. B. Djordjevic are with Department of Electrical and Computer Engineering, University of Arizona, Tucson, AZ 85721 USA (e-mail: arabaci@ece.arizona.edu; arabaci@email.arizona.edu; ivan@email. arizona.edu)

R. Saunders and R. M. Marcoccia are with Opnext, Inc., Los Gatos, CA 95032 USA (e-mail: rsaunders@opnext.com; rmarcoccia@opnext.com).

Color versions of one or more of the figures in this letter are available online at http://ieeexplore.ieee.org.

Digital Object Identifier 10.1109/LPT.2010.2040981
}

In our previous work [1], we showed how bit-interleaved low-density parity-check (LDPC)-coded modulation (BI-LDPC-CM) schemes can be used to achieve beyond $100-\mathrm{Gb} / \mathrm{s}$ transmission using commercially available components. In this letter, we propose using coded modulation based on nonbinary quasi-cyclic LDPC (QC-LDPC) codes to achieve the same task. QC-LDPC codes form an important class of linear block codes [2]. They can be encoded in linear time using simple shift-register-based architectures [2]. Also, the modular structure of their parity-check matrices can be exploited to ease hardware implementation of their decoders [3]. In addition these advantages, well-designed QC-LDPC codes have been shown to perform as good as random LDPC codes whose encoders/decoders tend to be quite complex.

Compared to BI-LDPC-CM, nonbinary-LDPC-coded-modulation (NB-LDPC-CM) offers several advantages: 1) $m$ binary LDPC encoders/decoders needed for multilevel modulation are collapsed into a single $2^{m}$-ary encoder/decoder (reducing the overall computational complexity of the system); 2) the use of block (de-)interleavers; binary-to-nonbinary and vice versa conversion interfaces are eliminated (reducing the complexity and latency in the system); 3) a posteriori probability (APP) demapper and LDPC decoder are integrated into a single block, and thus the need for iterating extrinsic information between APP demapper and LDPC decoder is eliminated (reducing the latency in the system). In addition to these advantages, we show that the proposed scheme provides higher coding gains than BI-LDPC-CM.

\section{Multilevel Coded-Modulation BASED ON NONBINARY LDPC CODES}

The transmitter $(\mathrm{Tx})$ and receiver $(\mathrm{Rx})$ configurations of the proposed NB-LDPC-CM scheme are shown in Fig. 1(a) and (b), respectively. At the Tx side, two independent data streams can be encoded using two different nonbinary LDPC codes of rates $R_{i}=K_{i} / N, i \in\{x, y\}$, where $K_{x}\left(K_{y}\right)$ denotes the number of information symbols corresponding to $\mathrm{x}-(\mathrm{y}-)$ polarization, and $N$ denotes the codeword length, which is the same for both LDPC codes. Hereafter, to simplify the notation, we use only $\mathrm{X}$-polarization in our description and note that the same concepts apply to the y-polarization. The component LDPC codes operate over finite fields $\operatorname{GF}\left(q=2^{m}\right)$. The Mapper accepts a nonbinary symbol from the LDPC encoder at time instance $l$ and determines the corresponding $q$-ary constellation point $\mathbf{s}_{l}=\left(I_{l}, Q_{l}\right)=\left|\mathbf{s}_{l}\right| \exp \left(j \phi_{l}\right)$ whose coordinates are used as the inputs for a dual-drive Mach-Zehnder modulator (MZM), where $I$ and $Q$ represent in-phase and quadrature components, respectively. The outputs of the MZMs are combined using a polarization beam combiner (PBC). The same distributed feedback (DFB) laser is used as a continuous-wave (CW) source with $\mathrm{x}$ - and $\mathrm{y}$-polarizations being separated by a polarization 


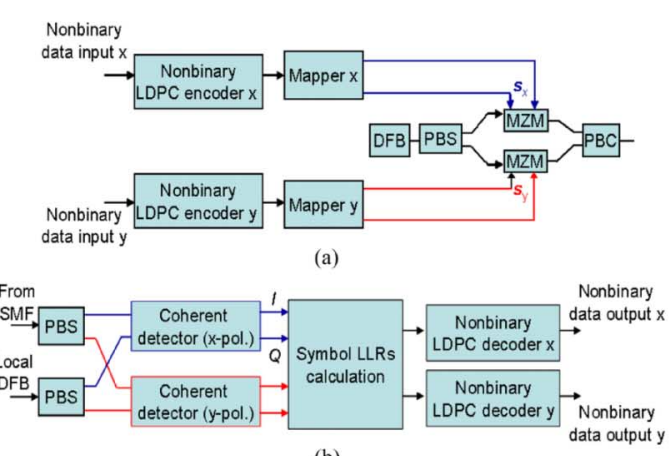

(b)

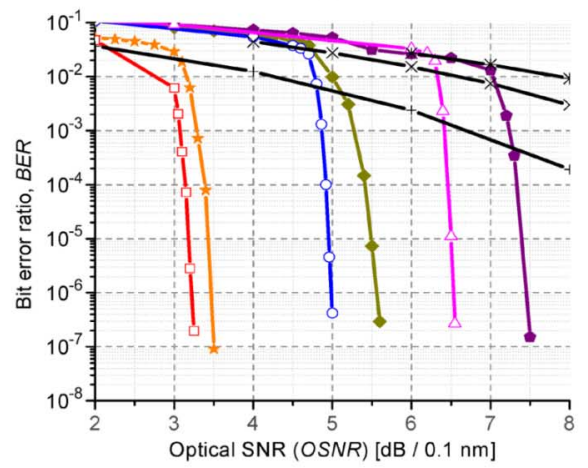

(d)
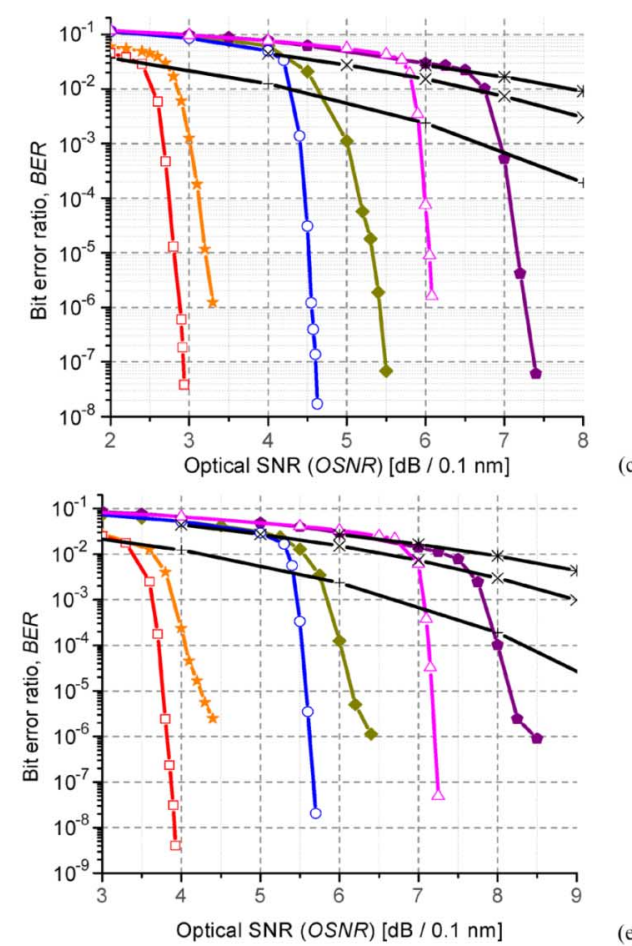

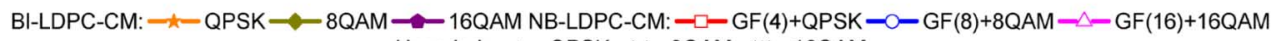
Uncoded: $-1-$ QPSK $-X-8 Q A M-*-16 Q A M$

Fig. 1. (a), (b) Configurations for the proposed NB-LDPC-CM scheme: (a) Tx and (b) Rx. (c)-(e) BER performance comparison between corresponding BI-LDPC-CM and NB-LDPC-CM schemes based on component codes at various code rates, $R:$ (c) $R=0.8,(\mathrm{~d}) R=0.85$, and (e) $R=0.9$. Note that $R_{s}=25 \mathrm{GS} / \mathrm{s}$. DFB laser: distributed feedback laser; PBS/PBC: polarization beam splitter and combiner; MZM: Mach-Zehnder modulator; LDPC code: low-density parity-check code; LLR: log-likelihood ratio.

beam splitter (PBS). Notice that for different polarizations we can use different constellations and different LDPC codes, but we keep the codeword lengths the same to simplify the synchronization.

At the Rx side, the $I$ - and $Q$-branches are sampled at the symbol rate. The symbol log-likelihood ratios (LLRs) are then calculated as follows:

$$
\lambda(\mathbf{s})=\log [P(\mathbf{r} \mid \mathbf{s}) P(\mathbf{s})] /\left[P\left(\mathbf{r} \mid \mathbf{s}_{0}\right) P\left(\mathbf{s}_{0}\right)\right]
$$

where $\mathbf{s}=\left(I_{l}, Q_{l}\right)$ and $\mathbf{r}=\left(r_{I}, r_{Q}\right)$ denote the transmitted constellation point and the received symbol at time instance $l$ (in either $\mathbf{x}$ - or $\mathbf{y}$-polarization), and $\mathbf{s}_{0}$ is the referent symbol. Note that the second equality follows from the Bayes' rule and that we determine $P(\mathbf{r} \mid \mathbf{s})$ via histograms. If the symbol rate is $R_{s}$ Giga-Symbols/s (GS/s), we can achieve $2 R_{s} \log _{2}(q)$ bits/s transmission using the proposed polarization-multiplexed NB-LDPC-CM scheme, where $q$ signifies the finite field order which is also equal to the constellation size of the modulation. For example, when $R_{s}=25 \mathrm{GS} / \mathrm{s}$, we can achieve 100-, 150-, and 200-Gb/s optical transmission using quadrature-phase-shift keying (QPSK), 8-quadrature amplitude modulation (QAM), and 16-QAM modulations, respectively.

\section{Evaluation of the Proposed Scheme}

In this section, we compare the performance of NB-LDPC-CM and BI-LDPC-CM schemes assuming amplified spontaneous emission (ASE) noise dominated scenario. To decode $q$-ary LDPC codes, the $q$-ary sum-product algorithm (SPA), i.e., QSPA [4], and its fast Fourier transform (FFT)-based variants can be used [4]-[6]. In this letter, we use the mixed-domain algorithm provided in [6], which we refer to as MD-FFT-QSPA. MD-FFT-QSPA operates in both real domain and logarithm domain to better exploit the benefits of each domain as elaborated on in [6]. When $q$ is a power of 2, the complex arithmetic operations in FFT-based QSPA can be avoided; thus, we focus only on the cases where $q=2^{m}$, for some positive integer $m$. Small cycles, particularly four-cycles, in the Tanner graphs of LDPC codes deteriorate the code performance under SPA decoding [2]. In this letter, to design NB-QC-LDPC codes without such small cycles, we followed a two-stage code construction technique which was discussed in detail in [7]. Using the parameters presented in Table I and the two-stage code construction technique, one can construct codes belonging to the same family of NB-QC-LDPC codes. In our floating-point simulations, for NB-LDPC-CM schemes, we used 50 decoding iterations in MD-FFT-QSPA. On the other hand, for BI-LDPC-CM schemes, we set the number of inner iterations in SPA to 25 and the number of global iterations between the APP demapper and binary LDPC decoders to 3. This indeed gives BI-LDPC-CM schemes an important advantage since they can take comparably more (total) number of iterations in decoding.

In Fig. 1(c)-(e), we compare the bit-error-ratio (BER) performances BI-LDPC-CM and NB-LDPC-CM schemes with polarization multiplexing. We tested both schemes using component codes at various code rates and modulation formats with various bandwidth efficiencies. (Exact parameters of the 
TABLE I

COMPLEXITY COMPARISON BETWEEN BI-LDPC-CM AND NB-LDPC-CM ${ }^{\dagger}$

\begin{tabular}{cccccc}
\hline \hline \multirow{2}{*}{$\begin{array}{l}\text { Code } \\
\text { Rate }\end{array}$} & $(\gamma, \rho)$ & $(N, K)$ & \multicolumn{3}{c}{ CR } \\
\cline { 4 - 6 } & & & QPSK & $\begin{array}{c}8 \\
\text { QAM }\end{array}$ & $\begin{array}{c}16 \\
\text { QAM }\end{array}$ \\
\hline 0.8 & $\begin{array}{l}(3,15) \\
(4,21)\end{array}$ & $\begin{array}{c}(8550,6840) \\
(8547,6922)\end{array}$ & 0.66 & 1.17 & 2.20 \\
\hline 0.85 & $\begin{array}{l}(3,20) \\
(4,27)\end{array}$ & $\begin{array}{l}(16200,13770) \\
(16200,13810)\end{array}$ & 0.65 & 1.15 & 2.16 \\
\hline 0.9 & $(3,29)$ & $(11832,10608)$ & 0.84 & 1.50 & 2.81 \\
\hline \hline
\end{tabular}

${ }^{\dagger}$ NB-LDPC-CM scheme is the first row of a given code rate.

TABLE II

CODING GAINS (DECIBEL) AT THE BER OF $10^{-6}$ FOR BI-LDPC-CM AND NB-LDPC-CM ${ }^{\dagger}$

\begin{tabular}{cccc}
\hline \hline Code & \multicolumn{3}{c}{ Constellation Size } \\
\cline { 2 - 4 } Rate & 4 & 8 & 16 \\
\hline \multirow{2}{*}{0.8} & 7.62 & 8.06 & 8.31 \\
& 7.19 & 7.21 & 7.11 \\
\hline \multirow{2}{*}{0.85} & 7.27 & 7.64 & 7.87 \\
& 7.02 & 7.06 & 6.94 \\
\hline \multirow{2}{*}{0.9} & 6.66 & 6.99 & 7.19 \\
& 6.00 & 6.24 & 5.96 \\
\hline \hline
\end{tabular}

${ }^{\dagger} \mathrm{NB}-\mathrm{LDPC}-\mathrm{CM}$ scheme is the first row of a given code rate.

component codes are given in Table I.) We can state with confidence that the proposed NB-LDPC-CM outperforms the BI-LDPC-CM at all the code rates regardless of the modulation format used. We calculated coding gains that both schemes offer for different modulations and tabulated them in Table II. As Table II reveals, the coding gain improvement that NB-LDPC-CM provides over the BI-LDPC-CM depends on the constellation size, and increases as the constellation size increases.

The slopes of the BER curves in Fig. 1 suggest that the performance gap between NB-LDPC-CM and BI-LDPC-CM increases as the optical signal-to-noise ratio (OSNR) in the system increases. We should note that if an error floor presents itself before the BER of interest, it can easily be eliminated by using a simple outer $\mathrm{BCH}$ or RS code of very high code rate [8]. Comparing the figures, we can clearly see the dramatic performance improvement that NB-LDPC-CM can bring to the forward-error correction (FEC) modules of future optical communication systems.

\section{COMPLEXITY ANALYSIS AND DISCUSSION}

Data processing at the check nodes is the dominant factor in the computational complexity of LDPC decoders; hence, we base our comparisons on this dominant factor. First, consider using the NB-LDPC-CM scheme based on a component $(\gamma, \rho)$-regular $2^{m}$-ary $\operatorname{LDPC}(N, K)$ code. For comparison, consider also using BI-LDPC-CM scheme employing $m$ identical $\left(\gamma_{b}, \rho_{b}\right)$-regular binary $\operatorname{LDPC}\left(N_{b}, K_{b}\right)$ codes as component codes. If we use MD-FFT-QSPA in the first case, the decoding complexity is given by the numerator in the complexity ratio (CR) term in (2). (For details on the complexity analysis of MD-FFT-QSPA, please see [6].) On the other hand, if we use Jacobian-based SPA [9] in the second case, the decoding complexity is given by the denominator in the $\mathrm{CR}$ term below

$$
\mathrm{CR}=\frac{2 \rho q M(m+1-1 /(2 \rho))}{15 m M_{b}\left(\rho_{b}-2\right)}
$$

where $M=N-K, M_{b}=N_{b}-K_{b}$, and $q=2^{m}$.

In Table I, we compare the complexity of corresponding NB-LDPC-CM and BI-LDPC-CM schemes employing component codes at various code rates and various modulation formats. When QPSK modulation is used, NB-LDPC-CM provides results in lower complexity systems but the additional coding gains that it offers over BI-LDPC-CM are not that high, i.e., in the ranges of $0.2-0.4 \mathrm{~dB}$ at the BER of $10^{-6}$ (see Table II). If we use 8-QAM modulation, the complexities become comparable; however, the additional coding gain that NB-LDPC-CM offers over BI-LDPC-CM becomes quite significant, i.e., $0.6-0.85 \mathrm{~dB}$ at the BER of $10^{-6}$. In the 16-QAM case, we observe that the complexity of NB-LDPC-CM is at least two times that of BI-LDPC-CM. On the other hand, NB-LDPC-CM provides impressive 0.9 - to 1.2 -dB coding gain improvement at the BER of $10^{-6}$ compared to BI-LDPC-CM, regardless of the component code rates. We should also note that error floors that appear in BI-LDPC-CM curves when component codes at the code rate $R=0.9$ are used can be avoided by using NB-LDPC-CM and nonbinary component codes. As opposed to doubling of the decoding complexity, reductions in latency, striking around 1-dB improvement in coding gain and resilience to error floors, can lead system designers to consider NB-LDPC-CM scheme even over much higher order fields to balance their link budgets.

\section{REFERENCES}

[1] I. B. Djordjevic, M. Cvijetic, L. Xu, and T. Wang, "Using LDPC-coded modulation and coherent detection for ultra high-speed optical transmission," J. Lightw. Technol., vol. 25, no. 11, pp. 3619-3625, Nov. 2007.

[2] S. Lin and D. J. Costello, Jr., Error Control Coding: Fundamentals and Applications, 2nd ed. Upper Saddle River, NJ: Prentice-Hall, 2004.

[3] Z. Wang and Z. Cui, "Low-complexity high-speed decoder design for quasi-cyclic LDPC codes," IEEE Trans. Very Large Scale Integr. (VLSI) Syst., vol. 15, no. 1, pp. 104-114, Jan. 2007.

[4] M. C. Davey, "Error-Correction Using Low-Density Parity-Check Codes," Ph.D. dissertation, University of Cambridge, Cambridge, U.K., 1999.

[5] D. Declercq and M. Fossorier, "Decoding algorithms for nonbinary LDPC codes over GF( $q$ ), IEEE Trans. Commun., vol. 55, no. 4, pp. 633-643, Apr. 2007.

[6] C. Spagnol, W. Marnane, and E. Popovici, "FPGA implementations of LDPC over GF $\left(2^{m}\right)$ decoders," in IEEE Workshop on Signal Proc. Syst., Shanghai, China, 2007, pp. 273-278.

[7] I. B. Djordjevic, M. Arabaci, and L. Minkov, "Next generation FEC for high-capacity communication in optical transport networks," J. Lightw. Technol., vol. 27, no. 16, pp. 3518-3530, Aug. 15, 2009.

[8] Y. Miyata, W. Matsumoto, H. Yoshida, and T. Mizuochi, "Efficient FEC for optical communications using concatenated codes to combat error-floor," presented at the Proc. OFC/NFOEC, San Diego, CA, 2008, Paper OTuE4.

[9] J. Chen, A. Dholakia, E. Eleftheriou, M. Fossorier, and X.-Y. Hu, "Reduced-complexity decoding of LDPC codes," IEEE Trans. Commun., vol. 53, no. 8, pp. 1288-1299, Jul. 2005. 